Proceedings of the 2007 Winter Simulation Conference

S. G. Henderson, B. Biller, M.-H. Hsieh, J. Shortle, J. D. Tew, and R. R. Barton, eds.

\title{
CONFIDENCE INTERVAL ESTIMATION USING LINEAR COMBINATIONS OF OVERLAPPING VARIANCE ESTIMATORS
}

\author{
Tûba Aktaran-Kalaycı \\ Department of Industrial Engineering \\ and Systems Engineering \\ University at Buffalo, SUNY \\ Buffalo, New York 14260, U.S.A.
}

\author{
David Goldsman \\ H. Milton Stewart School of \\ Industrial and Systems Engineering \\ Georgia Institute of Technology \\ Atlanta, GA 30332, U.S.A.
}

\author{
James R. Wilson \\ Edward P. Fitts Department of \\ Industrial and Systems Engineering \\ North Carolina State University \\ Raleigh, NC 27695-7906, U.S.A.
}

\begin{abstract}
We develop new confidence-interval estimators for the mean and variance parameter of a steady-state simulation output process. These confidence intervals are based on optimal linear combinations of overlapping estimators for the variance parameter. We present analytical and simulation-based results exemplifying the potential of this technique for improvements in accuracy for confidence intervals.
\end{abstract}

\section{INTRODUCTION}

Steady-state simulations can be used to analyze a variety of complicated systems. To complete a successful steady-state simulation study, one has to carry out a careful statistical analysis of the simulation's output. If the output process $\left\{Y_{i}: i \geq 1\right\}$ is in steady state, then, usually, we are interested in estimating the unknown mean $\mu$ of the process. The sample mean $\bar{Y}_{n} \equiv \sum_{i=1}^{n} Y_{i} / n$ is the usual estimator for $\mu$. But to complete the picture, the experimenter ought to estimate the sample mean's precision as well. The variance parameter,

$$
\sigma^{2} \equiv \lim _{n \rightarrow \infty} n \operatorname{Var}\left(\bar{Y}_{n}\right)
$$

is one of the measures of the sample mean's variability.

The objective of our research is to develop new confidence interval estimators for $\mu$ and $\sigma^{2}$. These new confidence intervals are based on forming an optimal linear combination of overlapping variance estimators (OLCOVE) (Aktaran-Kalayc1 2006, Aktaran-Kalayc1, Goldsman, and Wilson 2007). It has been shown that an OLCOVE can have lower bias and lower variance than those of the constituent estimators used to construct the linear combination. Using the OLCOVEs, we expect the corresponding confidence intervals to have better performance than certain existing estimators in the literature.

The organization of the remainder of this article is as follows. We first review in $\$ 2$ some background basics on OLCOVEs and standardized time series (STS). We approximate the distribution of an OLCOVE and then use this approximation to construct confidence intervals in $\$ 3$. Section 3 also provides Monte Carlo results that support our findings. Finally, we offer a summary in $\$ 4$.

\section{BACKGROUND}

\subsection{Setup for Using STS Variance Estimators}

We assume the stationary stochastic process $\left\{Y_{i}: i \geq 1\right\}$ has steady-state mean $\mu$ and variance parameter $\sigma^{2} \in(0, \infty)$ such that the following Functional Central Limit Theorem (FCLT) holds.

Assumption FCLT. For $n=1,2, \ldots$,

$$
X_{n}(t) \equiv \frac{\lfloor n t\rfloor\left(\bar{Y}_{\lfloor n t\rfloor}-\mu\right)}{\sigma \sqrt{n}} \text { for } t \in[0,1]
$$

satisfies

$$
X_{n} \underset{n \rightarrow \infty}{\Longrightarrow} \mathcal{W}
$$




\section{Aktaran-Kalaycı, Goldsman, and Wilson}

where: $\lfloor\cdot\rfloor$ denotes the greatest integer function; $\mathcal{W}$ is a standard Brownian motion process on $[0,1]$; and $\underset{n \rightarrow \infty}{\Longrightarrow}$

denotes weak convergence in $D[0,1]$, the associated probability space of functions on $[0,1]$ that are rightcontinuous with left-hand limits, as $n \rightarrow \infty$. See also Billingsley (1968).

Suppose we have $n$ observations $Y_{1}, Y_{2}, \ldots, Y_{n}$ on hand, and we form $n-m+1$ overlapping batches, each of size $m$. In particular, the observations $Y_{i}, Y_{i+1}, \ldots, Y_{i+m-1}$ constitute batch $i$, for $i=1,2, \ldots, n-m+1$. Similar to the original definition in Schruben (1983), the standardized time series from overlapping batch $i$ is

$$
T_{i, m}^{\mathrm{O}}(t) \equiv \frac{\lfloor m t\rfloor\left(\bar{Y}_{i, m}^{\mathrm{O}}-\bar{Y}_{i,\lfloor m t\rfloor}^{\mathrm{O}}\right)}{\sigma \sqrt{m}} \text { for } 0 \leq t \leq 1
$$

where $\bar{Y}_{i, j}^{\mathrm{O}} \equiv \sum_{\ell=0}^{j-1} Y_{i+\ell} / j$ for $i=1,2, \ldots, n-m+1$ and $j=1,2, \ldots, m$. Alexopoulos et al. (2006) show that, under Assumption FCLT,

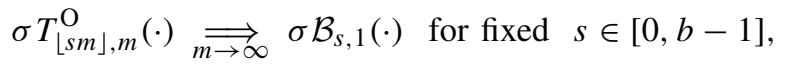

where $b \equiv n / m>1$ is a fixed ratio; and for $r \in[1, b)$ and $s \in[0, b-r]$, we let $\mathcal{B}_{s, r}(\cdot)$ denote a Brownian bridge process on the unit interval,

$$
\mathcal{B}_{s, r}(t)=\frac{t[\mathcal{W}(s+r)-\mathcal{W}(s)]-[\mathcal{W}(s+t r)-\mathcal{W}(s)]}{\sqrt{r}},
$$

for $t \in[0,1]$. Moreover, we also have the following useful asymptotic properties as $n \rightarrow \infty$ :

$$
\begin{aligned}
& \sqrt{n}\left(\bar{Y}_{n}-\mu\right) \sim \sigma \operatorname{Nor}(0,1) ; \text { and } \\
& \sqrt{n}\left(\bar{Y}_{n}-\mu\right) \text { and } \sigma T_{1, n}^{\mathrm{O}} \text { are independent. }
\end{aligned}
$$

\subsection{Overlapping Area Variance Estimator}

The idea is to form a separate estimator for $\sigma^{2}$ from each overlapping batch - even though the resulting estimators are clearly dependent-and then to average those estimators. In the context of this article, the fixed quantity $b$ may in general take any real value exceeding one while we let the batch size $m \rightarrow \infty$; and thus the total sample size is always taken to be $\lfloor b m\rfloor$. The STS area estimator from the $i$ th overlapping batch is

$$
A_{i}^{\mathrm{O}}(f ; m) \equiv\left[\frac{1}{m} \sum_{\ell=1}^{m} f(\ell / m) \sigma T_{i, m}^{\mathrm{O}}(\ell / m)\right]^{2},
$$

for $i=1,2, \ldots,\lfloor b m\rfloor-m+1$, where $f(t)$ is a weighting function that has a continuous second derivative $\left(\mathrm{d}^{2} / \mathrm{d} t^{2}\right) f(t)$ on the unit interval $[0,1]$ and that satisfies the normalization condition $\operatorname{Var}\left(\int_{0}^{1} f(t) \mathcal{B}_{0,1}(t) \mathrm{d} t\right)=1$. Averaging, we have the overlapping area estimator (OAE) for $\sigma^{2}$

$$
\mathcal{A}^{\mathrm{O}}(f ; b, m) \equiv \frac{1}{\lfloor b m\rfloor-m+1} \sum_{i=1}^{\lfloor b m\rfloor-m+1} A_{i}^{\mathrm{O}}(f ; m)
$$

Alexopoulos et al. (2006) show that

$$
\begin{aligned}
\mathcal{A}^{\mathrm{O}}(f ; b, m) \underset{m \rightarrow \infty}{\Longrightarrow} & \mathcal{A}^{\mathrm{O}}(f ; b) \equiv \\
& \frac{\sigma^{2}}{b-1} \int_{0}^{b-1}\left[\int_{0}^{1} f(t) \mathcal{B}_{s, 1}(t) \mathrm{d} t\right]^{2} \mathrm{~d} s .
\end{aligned}
$$

The next theorem gives the expected value of the OAE. Before that we define the following quantities: $R_{\ell} \equiv \operatorname{Cov}\left(Y_{1}, Y_{1+\ell}\right)$ for $\ell=1,2, \ldots$;

$$
\gamma \equiv-2 \sum_{\ell=1}^{\infty} \ell R_{\ell}
$$

(Song, and Schmeiser 1995);

$$
F(t) \equiv \int_{0}^{t} f(s) \mathrm{d} s, \quad \bar{F}(t) \equiv \int_{0}^{t} F(s) \mathrm{d} s
$$

for $0 \leq t \leq 1$; and

$$
F^{\star} \equiv\left[(F(1)-\bar{F}(1))^{2}+\bar{F}^{2}(1)\right] / 2
$$

Theorem 1 (See, e.g., Foley and Goldsman 1999.) Suppose that $\left\{Y_{i}: i \geq 1\right\}$ is a stationary process for which Assumption FCLT holds and $\sum_{\ell=1}^{\infty} \ell^{2}\left|R_{\ell}\right|<\infty$. Further, suppose that the family of random variables $\left\{\left[\mathcal{A}^{\mathrm{O}}(f ; b, m)\right]^{2}: m=1,2, \ldots\right\}$ is uniformly integrable (cf. Billingsley 1968). If $f(\cdot)$ satisfies the above-mentioned smoothness and normalization requirements, then

$$
\mathrm{E}\left[\mathcal{A}^{\mathrm{O}}(f ; b, m)\right]=\sigma^{2}+\frac{F^{\star} \gamma}{m}+o\left(\frac{1}{m}\right) \text { as } m \rightarrow \infty \text {. }
$$

Calculation of the asymptotic variance of the OAE can be performed using Eq. (5) as described in Alexopoulos et al. (2006), who show that as $m \rightarrow \infty$ the variance of the OAE depends on the choice of the function $f(\cdot)$. In fact, all the OAEs discussed subsequently have smaller limiting variances than those of the following:

(a) the benchmark nonoverlapping batch means variance estimator, whose variance is $2 \sigma^{4} /(b-1)$ (see Chien, Goldsman, and Melamed 1997); 
(b) the overlapping batch means variance estimator, whose variance is approximately $\frac{4}{3} \sigma^{4} / b$ (see Meketon and Schmeiser 1984); and

(c) the nonoverlapping version of the area estimator with any legal weighting function, whose variance is $2 \sigma^{4} / b$ (see Goldsman, Meketon, and Schruben 1990).

Example 2 Schruben (1983) studied the nonoverlapping version of the area estimator with $f_{0}(t) \equiv \sqrt{12}$ for $0 \leq t \leq 1$. For the overlapping version, Theorem 1 yields $\mathrm{E}\left[\mathcal{A}^{\mathrm{O}}\left(f_{0} ; b, m\right)\right]=\sigma^{2}+3 \gamma / m+o(1 / m)$. Further, as $m \rightarrow \infty$, some algebra involving Eq. (5) gives the asymptotic variance

$$
\operatorname{Var}\left(\mathcal{A}^{\mathrm{O}}\left(f_{0} ; b\right)\right)=\frac{24 b-31}{35(b-1)^{2}} \sigma^{4} \text { for } b \geq 2 .
$$

If one chooses weights having $F^{\star}=0$, then the resulting OAE is first-order unbiased for $\sigma^{2}$, i.e., its bias is $o(1 / m)$. Such an example is $f_{2}(t) \equiv \sqrt{840}\left(3 t^{2}-3 t+1 / 2\right)$ for $0 \leq t \leq 1$ (see Goldsman, Meketon, and Schruben 1990, Goldsman and Schruben 1990); and for this weighting function, the asymptotic variance as $m \rightarrow \infty$ is

$$
\operatorname{Var}\left(\mathcal{A}^{\mathrm{O}}\left(f_{2} ; b\right)\right)=\frac{3514 b-4359}{4290(b-1)^{2}} \sigma^{4} \text { for } b \geq 2 \text {. }
$$

\subsection{Optimal Linear Combination of Overlapping Area Estimators}

For the OLCOVEs, we calculate $k$ OAEs, $\mathcal{A}^{\mathrm{O}}\left(f ; B_{1}, M_{1}\right)$, $\mathcal{A}^{\mathrm{O}}\left(f ; B_{2}, M_{2}\right), \ldots, \mathcal{A}^{\mathrm{O}}\left(f ; B_{k}, M_{k}\right)$, where we use the same data over a variety of different integer batch sizes, $M_{1}, M_{2}$, $\ldots, M_{k}$. Specifically, for $j=1,2, \ldots, k$, we let $r_{j} \in[1, \infty)$ and take $M_{j} \equiv\left\lfloor r_{j} m\right\rfloor$ as the $j$ th batch size, with the corresponding quantity $B_{j} \equiv\lfloor b m\rfloor / M_{j}$ so that the total sample size is $B_{j} M_{j}=\lfloor b m\rfloor$, and $B_{j}$ is the associated samplesize-to-batch-size ratio. Then we form a linear combination of these $k$ estimators and scale appropriately. We use standard regression techniques underlying the method of control variates (Lavenberg and Welch 1981) to determine scaling factors that preserve low bias and minimize variance.

$$
\text { Let } \boldsymbol{M} \equiv\left[M_{1}, M_{2}, \ldots, M_{k}\right] \text { and } \boldsymbol{B} \equiv
$$
$\left[B_{1}, B_{2}, \ldots, B_{k}\right] . \quad$ Let $\boldsymbol{\alpha} \equiv\left[\alpha_{1}, \alpha_{2}, \ldots, \alpha_{k-1}\right]$ denote the vector of weights used to form the linear combination

$$
\begin{aligned}
& \mathcal{A}^{\mathrm{LO}}(f ; \boldsymbol{B}, \boldsymbol{M}, \boldsymbol{\alpha}) \\
& \quad \equiv \sum_{i=1}^{k-1} \alpha_{i} \mathcal{A}^{\mathrm{O}}\left(f ; B_{i}, M_{i}\right)+\left(1-\sum_{i=1}^{k-1} \alpha_{i}\right) \mathcal{A}^{\mathrm{O}}\left(f ; B_{k}, M_{k}\right)
\end{aligned}
$$

Theorem 3 (Aktaran-Kalayc1 2006) Let $\boldsymbol{r} \equiv\left[r_{1}, r_{2}\right.$, $\left.\ldots, r_{k}\right]$ denote the vector of batch-size multipliers. If $b>$ $\max \left\{r_{j}: j=1, \ldots, k\right\}$, then let $\mathcal{A}^{\mathrm{O}}(f ; \boldsymbol{B}, \boldsymbol{r}, \boldsymbol{M})$ denote the $k \times 1$ vector whose $j$ th component is $\mathcal{A}^{\mathrm{O}}\left(f ; B_{j}, M_{j}\right)$ for $j=1, \ldots, k$. Similarly, let $\mathcal{A}^{\mathrm{O}}(f ; b, \boldsymbol{r})$ denote the $k \times 1$ vector whose $j$ th component is

$$
\mathcal{A}_{j}^{\mathrm{O}}\left(f ; b, r_{j}\right) \equiv\left(b-r_{j}\right)^{-1} \int_{0}^{b-r_{j}}\left[\sigma \int_{0}^{1} f(t) \mathcal{B}_{s, r_{j}}(t) \mathrm{d} t\right]^{2} \mathrm{~d} s
$$

for $j=1, \ldots, k$. If Assumption FCLT holds and $f(t)$ is normalized as discussed in Section 2.2, then

$$
\mathcal{A}^{\mathrm{O}}(f ; \boldsymbol{B}, \boldsymbol{r}, \boldsymbol{M}) \underset{m \rightarrow \infty}{\Longrightarrow} \mathcal{A}^{\mathrm{O}}(f ; b, \boldsymbol{r})
$$

We calculate the asymptotic covariances of the OAEs based on different batch sizes using Eq. (8) as described in Aktaran-Kalayc1, Goldsman, and Wilson (2007). Then we compute the variance-optimizing coefficients in the linear combination, $\boldsymbol{\alpha}^{\star}$, based on these covariances. Table 1 presents properties of various OLCOVEs based on different batch size sets $\boldsymbol{M}$. We see that the estimator has bias of the form $\gamma c / m$, where the constant $c$ decreases from 3.00 to 2.59 as we add more and more terms (up to 20) to the linear combination; the estimator $\mathcal{A}^{\mathrm{LO}}\left(f_{2} ; \boldsymbol{B}, \boldsymbol{M}, \boldsymbol{\alpha}^{\star}\right)$ only has $o(1 / m)$ bias. Further, in this example, the standardized variance of $\mathcal{A}^{\mathrm{LO}}\left(f_{0} ; \boldsymbol{B}, \boldsymbol{M}, \boldsymbol{\alpha}^{\star}\right)$ decreases from 0.686 to 0.610 (about a $12 \%$ reduction) as we add more terms, while that of $\mathcal{A}^{\mathrm{LO}}\left(f_{2} ; \boldsymbol{B}, \boldsymbol{M}, \boldsymbol{\alpha}^{\star}\right)$ decreases from 0.819 to 0.688 (about a $16 \%$ reduction).

Table 1: Approximate asymptotic bias and variance of various OLCOVEs $\mathcal{A}^{\mathrm{LO}}\left(f ; \boldsymbol{B}, \boldsymbol{M}_{k}, \boldsymbol{\alpha}^{\star}\right)$ (where $\boldsymbol{M}_{k} \equiv$ $[m, 2 m, \ldots, k m])$ as $m \rightarrow \infty$.

\begin{tabular}{ccc|cc}
\hline & \multicolumn{2}{c}{$f_{0}$} & \multicolumn{2}{c}{$f_{2}$} \\
\cline { 2 - 5 }$k$ & $\frac{m}{\gamma}$ Bias & $\frac{b}{\sigma^{4}} \operatorname{Var}$ & $\frac{m}{\gamma}$ Bias & $\frac{b}{\sigma^{4}} \operatorname{Var}$ \\
\hline 1 & 3.00 & 0.686 & $o(1)$ & 0.819 \\
2 & 2.77 & 0.663 & $o(1)$ & 0.782 \\
3 & 2.71 & 0.638 & $o(1)$ & 0.731 \\
4 & 2.67 & 0.630 & $o(1)$ & 0.722 \\
5 & 2.64 & 0.625 & $o(1)$ & 0.710 \\
10 & 2.61 & 0.615 & $o(1)$ & 0.695 \\
20 & 2.59 & 0.610 & $o(1)$ & 0.688 \\
\hline
\end{tabular}

\section{DENSITY AND CONFIDENCE-INTERVAL ESTIMATION}

To estimate the probability density functions (p.d.f.'s) of the estimators, we generated 1,000,000 independent sample paths from an i.i.d. standard normal process, where each path consists of 10,240 observations with $m=512$ and $b=20$. Note that these settings are large enough for approximate convergence of the estimators to their limiting distributions. From these sample paths we computed the nonoverlapping, overlapping $\left(\mathcal{A}^{\mathrm{O}}(f ; b, m)\right)$, and OLCOVE 
$\left(\mathcal{A}^{\mathrm{LO}}\left(f ; \boldsymbol{B}, \boldsymbol{M}_{5}, \boldsymbol{\alpha}^{\star}\right)\right)$ versions of area estimators, where $\boldsymbol{M}_{5}=[m, \ldots, 5 m]$.

From the computed estimates, we obtained the empirical p.d.f.'s. Superimposing the empirical p.d.f.'s of estimators gives Figure 1. The figure shows that the OLCOVEs have lighter tails (i.e., lower variance) than the underlying OAEs, and the OAEs have lighter tails than do their nonoverlapping counterparts.

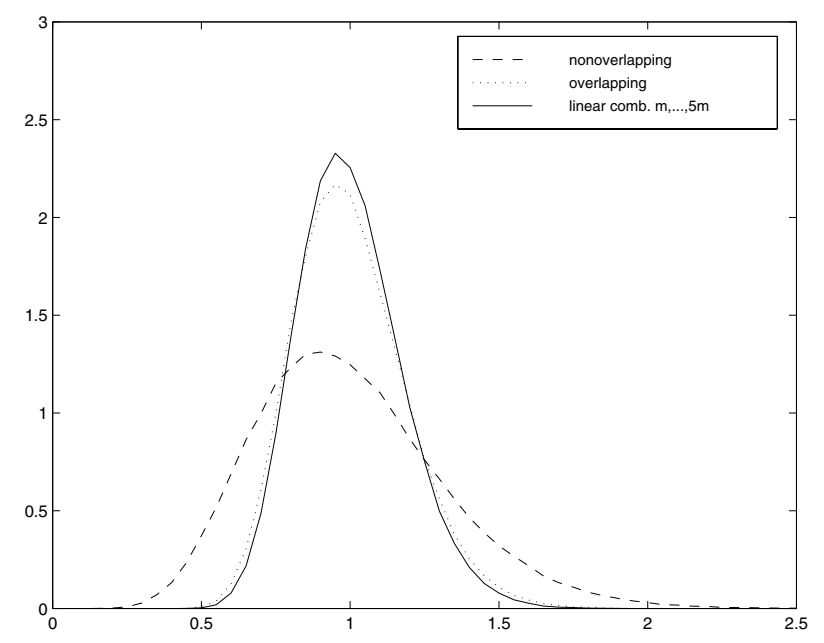

(a) Unweighted area estimators

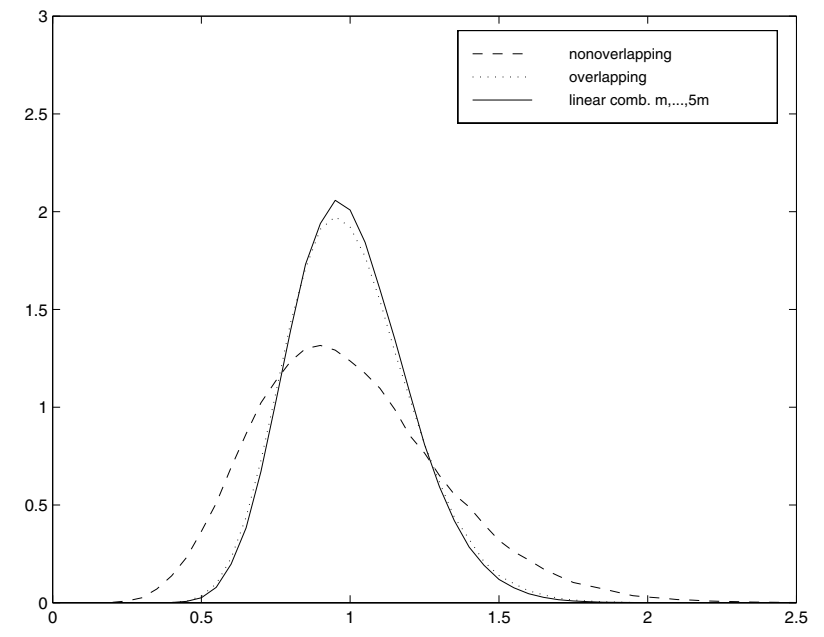

(b) Area estimators with quadratic weights $f_{2}$

Figure 1: Estimated p.d.f.'s for STS area variance estimators based on 1,000,000 sample paths of the i.i.d. standard normal process with $m=512$ and $b=20$.

Letting $\dot{\sim}$ denote the phrase "is approximately distributed as," we adopt the approximation technique of Satterthwaite (1941) to obtain

$$
\mathcal{V}(b, m) \dot{\sim} \mathrm{E}[\mathcal{V}(b, m)] \chi_{v_{\text {eff }}}^{2} / \nu_{\text {eff }},
$$

where $\mathcal{V}(b, m)$ is a generic variance estimator and the quantity $v_{\text {eff }}$ is called the effective degrees of freedom (d.o.f.) and is given by

$$
v_{\mathrm{eff}}=\llbracket 2 \mathrm{E}^{2}[\mathcal{V}(b, m)] / \operatorname{Var}(\mathcal{V}(b, m)) \rrbracket .
$$

Using (9) and (10), we calculate the effective d.o.f. for various OLCOVEs, with $\boldsymbol{M}_{k}=[m, \ldots, k m]$ and the $j$ th element of $\boldsymbol{B}$ defined as $B_{j}=\lfloor b m\rfloor /(j m)$ for $j=1, \ldots, k$. The results are given in Table 2 for the various OLCOVEs under consideration here. From (5) we see that $\sigma^{2}$ simply serves as a scale parameter in the limiting distribution of $\mathcal{A}^{\mathrm{O}}(f ; b, m)$ as $m \rightarrow \infty$; and thus the formula (10) for the effective d.o.f. applies asymptotically to all stochastic processes satisfying Assumption FCLT as $m \rightarrow \infty$.

Table 2: Effective degrees of freedom $v_{\text {eff }}$ for various OLCOVEs with $b=20$ for all processes satisfying Assumption FCLT as $m \rightarrow \infty$.

\begin{tabular}{c|ccccc}
\hline & \multicolumn{6}{|c}{ Effective Degrees of Freedom } \\
Estimator & $\boldsymbol{M}_{1}$ & $\boldsymbol{M}_{2}$ & $\boldsymbol{M}_{3}$ & $\boldsymbol{M}_{4}$ & $\boldsymbol{M}_{5}$ \\
\hline $\mathcal{A}^{\mathrm{LO}}\left(f_{0} ; \boldsymbol{B}, \boldsymbol{M}, \boldsymbol{\alpha}^{\star}\right)$ & 56 & 58 & 60 & 60 & 61 \\
$\mathcal{A}^{\mathrm{LO}}\left(f_{2} ; \boldsymbol{B}, \boldsymbol{M}, \boldsymbol{\alpha}^{\star}\right)$ & 47 & 49 & 52 & 53 & 53 \\
\hline
\end{tabular}

To illustrate the accuracy of our approximations to the limiting p.d.f.'s of the variance estimators, Figure 2 superimposes the empirical p.d.f.'s of the OLCOVEs and the corresponding fitted p.d.f.'s based on the approximation (9) and (10) (using $v_{\text {eff }}$ from Table 2),

$$
\mathcal{A}^{\mathrm{LO}}\left(f ; \boldsymbol{B}, \boldsymbol{M}, \boldsymbol{\alpha}^{\star}\right) \dot{\sim} \sigma^{2} \chi_{v_{\mathrm{eff}}}^{2} / v_{\mathrm{eff}}
$$

Figure 2 shows that we have very good approximations to the target p.d.f.'s. This finding immediately suggests that we can use OLCOVEs to construct approximately valid confidence intervals for the parameters $\mu$ and $\sigma^{2}$.

\subsection{Confidence Intervals for $\mu$}

It follows from the properties given in Eq.s (3), (4), and (11) that

$$
\frac{\bar{Y}_{n}-\mu}{\sqrt{\mathcal{A}^{\mathrm{LO}}\left(f ; \boldsymbol{B}, \boldsymbol{M}, \boldsymbol{\alpha}^{\star}\right) / n}} \dot{\sim} t_{v_{\mathrm{eff}}},
$$

for sufficiently large $m$, where $t_{v}$ denotes a Student's $t$ random variable with $v$ d.o.f. Then an approximate 100(1$\beta) \%$ confidence interval for $\mu$ is given by

$$
\bar{Y}_{n} \pm t_{1-\beta / 2, v_{\mathrm{eff}}} \sqrt{\mathcal{A}^{\mathrm{LO}}\left(f ; \boldsymbol{B}, \boldsymbol{M}, \boldsymbol{\alpha}^{\star}\right) / n}
$$


Aktaran-Kalaycı, Goldsman, and Wilson

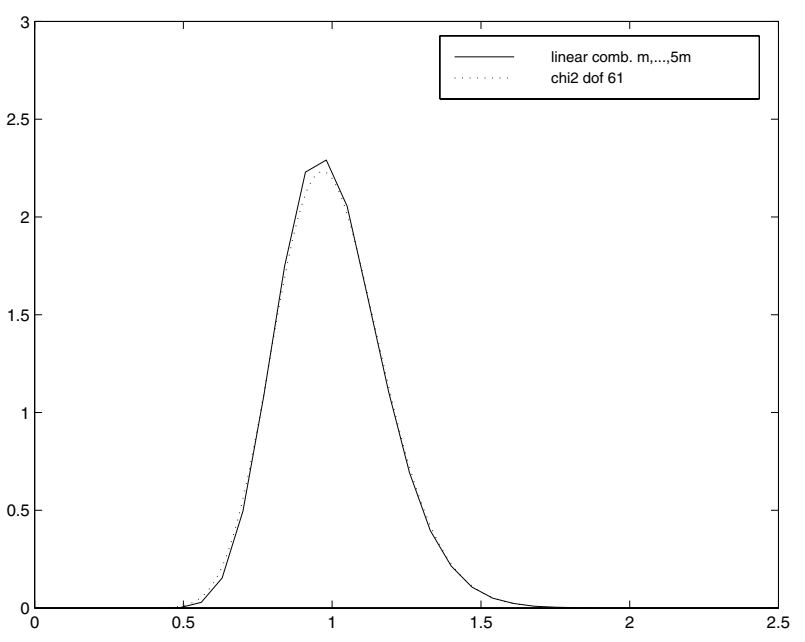

(a) Unweighted area estimators

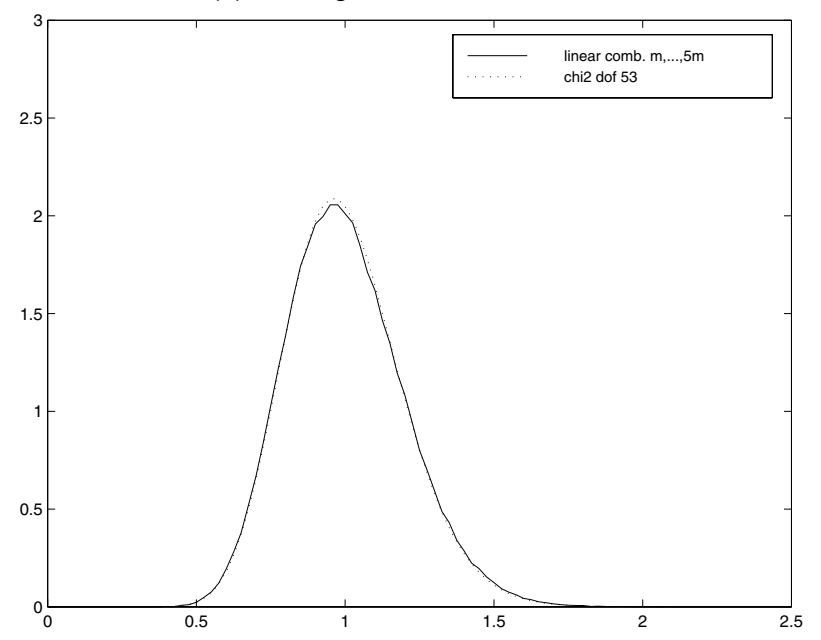

(b) Area estimators with quadratic weights $f_{2}$

Figure 2: Estimated and fitted p.d.f.'s for various OLCOVEs based on 1,000,000 sample paths of the i.i.d. standard normal process with $m=512$, and $b=20$.

where $t_{\omega, v}$ denotes the $\omega$-quantile of a Student's $t$ random variable with $v$ d.o.f.

Example 4 We use 1,000,000 independent sample paths of a first-order autoregressive [AR(1)] process to compute $1,000,000$ realizations of $\bar{Y}_{n}$ and various OLCOVEs. The specific AR(1) process is given by $Y_{i}=\phi Y_{i-1}+\epsilon_{i}$, $i=1,2, \ldots$, where the $\epsilon_{i}$ 's are i.i.d. $\operatorname{Nor}\left(0,1-\phi^{2}\right), Y_{0}$ is initialized as a standard normal random variate, independently of the $\epsilon_{i}$ 's, and $-1<\phi<1$. For the current experiment, we take $\phi=0.9$, a value that yields a moderately highly autocorrelated stationary process. We construct $1,000,000$ two-sided $90 \%$ confidence intervals for $\mu$ from Eq. (13), where the corresponding value $v_{\text {eff }}$ for the effective degrees of freedom is given in Table 2. Then we estimate the coverage probabilities for the two-sided $90 \%$ confidence intervals by determining the proportion of the confidence
Table 3: Estimated coverage probabilities of two-sided 90\% confidence intervals for $\mu$ from various OLCOVEs based on $1,000,000$ sample paths of the AR(1) process with $\phi=0.9, m=1024$, and $b=20$.

\begin{tabular}{c|cc}
\hline & $\mathcal{A}^{\mathrm{LO}}\left(f_{0} ; \boldsymbol{B}, \boldsymbol{M}, \boldsymbol{\alpha}^{\star}\right)$ & $\mathcal{A}^{\mathrm{LO}}\left(f_{2} ; \boldsymbol{B}, \boldsymbol{M}, \boldsymbol{\alpha}^{\star}\right)$ \\
\hline $\boldsymbol{M}_{1}$ & 0.8958 & 0.8997 \\
$\boldsymbol{M}_{2}$ & 0.8961 & 0.8997 \\
$\boldsymbol{M}_{3}$ & 0.8960 & 0.8999 \\
$\boldsymbol{M}_{4}$ & 0.8962 & 0.8998 \\
$\boldsymbol{M}_{5}$ & 0.8962 & 0.8999 \\
\hline
\end{tabular}

intervals that actually contain $\mu=0$. These results are presented in Table 3.

From Table 3, we observe that the achieved empirical coverage probabilities do not differ substantially from the targeted coverage probability, 0.90, thus indicating that the confidence interval procedure works for large-enough batch size $m$.

\subsection{Confidence Intervals for $\sigma^{2}$}

Assuming $\mathrm{E}\left[\mathcal{A}^{\mathrm{LO}}\left(f ; \boldsymbol{B}, \boldsymbol{M}, \boldsymbol{\alpha}^{\star}\right)\right]=\sigma^{2}$ and using Eq. (11), we see that an approximate $100(1-\beta) \%$ confidence interval for $\sigma^{2}$ is given by

$$
\frac{\nu_{\text {eff }} \mathcal{A}^{\mathrm{LO}}\left(f ; \boldsymbol{B}, \boldsymbol{M}, \boldsymbol{\alpha}^{\star}\right)}{\chi_{1-\beta / 2, \nu_{\text {eff }}}^{2}} \leq \sigma^{2} \leq \frac{\nu_{\mathrm{eff}} \mathcal{A}^{\mathrm{LO}}\left(f ; \boldsymbol{B}, \boldsymbol{M}, \boldsymbol{\alpha}^{\star}\right)}{\chi_{\beta / 2, v_{\mathrm{eff}}}^{2}},
$$

where $\chi_{\omega, \nu}^{2}$ denotes the $\omega$-quantile of the $\chi^{2}$ distribution with $v$ degrees of freedom.

Example 5 We use the OLCOVEs computed from the $1,000,000$ independent sample paths of the AR(1) process from Example 4 to construct two-sided $90 \%$ confidence intervals for $\sigma^{2}$. The two-sided confidence intervals for $\sigma^{2}$ are given by Eq. (14), where the corresponding $v_{\text {eff }}$ is from Table 2. We obtain the estimated coverage probabilities for the two-sided $90 \%$ confidence intervals as presented in Table 4.

Table 4: Estimated coverage probabilities of two-sided $90 \%$ confidence intervals for $\sigma^{2}$ from various OLCOVEs based on 1,000,000 sample paths of the AR(1) process with $\phi=0.9, m=1024$, and $b=20$.

\begin{tabular}{l|cc}
\hline & $\mathcal{A}^{\mathrm{LO}}\left(f_{0} ; \boldsymbol{B}, \boldsymbol{M}, \boldsymbol{\alpha}^{\star}\right)$ & $\mathcal{A}^{\mathrm{LO}}\left(f_{2} ; \boldsymbol{B}, \boldsymbol{M}, \boldsymbol{\alpha}^{\star}\right)$ \\
\hline $\boldsymbol{M}_{1}$ & 0.8697 & 0.8891 \\
$\boldsymbol{M}_{2}$ & 0.8708 & 0.8895 \\
$\boldsymbol{M}_{3}$ & 0.8706 & 0.8902 \\
$\boldsymbol{M}_{4}$ & 0.8730 & 0.8887 \\
$\boldsymbol{M}_{5}$ & 0.8719 & 0.8906 \\
\hline
\end{tabular}

In obtaining Eq. (14), we have assumed that all the estimators are unbiased for $\sigma^{2}$. However, we know that 
$\mathcal{A}^{\mathrm{LO}}\left(f_{0} ; \boldsymbol{B}, \boldsymbol{M}, \boldsymbol{\alpha}^{\star}\right)$ is moderately biased. Thus, our assumption causes the coverage probabilities to be a bit off for the $\mathcal{A}^{\mathrm{LO}}\left(f_{0} ; \boldsymbol{B}, \boldsymbol{M}, \boldsymbol{\alpha}^{\star}\right)$ entries in Table 4 . On the other hand, the empirical coverage probabilities do not differ substantially from the targeted coverage probability for the $\mathcal{A}^{\mathrm{LO}}\left(f_{2} ; \boldsymbol{B}, \boldsymbol{M}, \boldsymbol{\alpha}^{\star}\right)$ estimators.

\section{SUMMARY AND RECOMMENDATIONS}

The goal of this paper has been to study a new class of confidence interval estimators for $\mu$ and $\sigma^{2}$ arising from a stationary simulation output process. The new estimators are based on linear combinations of overlapping estimators, where each constituent of the linear combination uses a different batch size.

We have shown that the distributions of the OLCOVEs can be approximated quite accurately by scaled $\chi^{2}$ distributions with appropriate degrees of freedom (at least for the specific AR(1) process we considered). We have applied this approximation to construct confidence intervals for the parameters $\mu$ and $\sigma^{2}$ based on various OLCOVEs; and we have conducted Monte Carlo studies to see how these confidence intervals perform when applied to simple stochastic processes. For the confidence intervals for $\mu$, we found that the achieved coverage probability is practically the same as the targeted coverage probability (for large enough batch sizes). Regarding the confidence intervals for $\sigma^{2}$, we found that actual coverage probabilities based on first-order unbiased estimators of $\sigma^{2}$ do not differ substantially from the targeted coverage probabilities for batch sizes that are large enough.

\section{REFERENCES}

Aktaran-Kalayc1, T. 2006. Steady-state analyses: Variance estimation in simulations and dynamic pricing in service systems. Doctoral dissertation, H. Milton Stewart School of Industrial and Systems Engineering, Georgia Institute of Technology, Atlanta, Georgia. Available via $<$ hdl. handle.net/1853/13993 $>$ [accessed June 18, 2007].

Aktaran-Kalayc1, T., and D. Goldsman. 2005. Linear combinations of overlapping variance estimators for simulations. In Proceedings of the 2005 Winter Simulation Conference, ed. M. E. Kuhl, N. M. Steiger, F. B. Armstrong, and J. A. Joines, 756-762. Piscataway, New Jersey: Institute of Electrical and Electronics Engineers. <www. informs-sim.org/wsc0 5papers / 087. . pdf> [accessed June 24, 2007].

Aktaran-Kalayc1, T., D. Goldsman, and J. R. Wilson. 2007. Linear combinations of overlapping variance estimators for simulation. Operations Research Letters 35:439447.
Alexopoulos, C., N. T. Argon, D. Goldsman, G. Tokol, and J. R. Wilson. 2006. Overlapping variance estimators for simulation. Operations Research, to appear. Available online via <ftp.ncsu.edu/pub/ eos/pub/jwilson/ovestv72.pdf> [accessed June 14, 2007].

Billingsley, P. 1968. Convergence of probability measures. New York: Wiley.

Chien, C., D. Goldsman, and B. Melamed. 1997. Largesample results for batch means. Management Science 43:1288-1295.

Foley, R. D., and D. Goldsman. 1999. Confidence intervals using orthonormally weighted standardized time series. ACM Transactions on Modeling and Computer Simulation 9:297-325.

Goldsman, D., M. Meketon, and L. Schruben. 1990. Properties of standardized time series weighted area variance estimators. Management Science 36:602-612.

Goldsman, D., and L. Schruben. 1990. New confidence interval estimators using standardized time series. Management Science 36:393-397.

Lavenberg, S. S., and P. D. Welch. 1981. A perspective on the use of control variables to increase the efficiency of Monte Carlo simulations. Management Science 27:322335.

Meketon, M. S., and B. W. Schmeiser. 1984. Overlapping batch means: Something for nothing? In Proceedings of the 1984 Winter Simulation Conference, ed. S. Sheppard, U. Pooch, and D. Pegden, 227-230. Piscataway, New Jersey: Institute of Electrical and Electronics Engineers.

Satterthwaite, F. E. 1941. Synthesis of variance. Psychometrika 6 (5): 309-316.

Schruben, L.. 1983. Confidence interval estimation using standardized time series. Operations Research 31:10901108.

Song, W. T., and B. W. Schmeiser. 1995. Optimal meansquared-error batch sizes. Management Science 41:110123.

\section{AUTHOR BIOGRAPHIES}

TÛBA AKTARAN-KALAYCI is an Assistant Professor in the Department of Industrial and Systems Engineering at the University at Buffalo. She received her Ph.D. from the H. Milton Stewart School of Industrial and Systems Engineering in the Georgia Institute of Technology. Her research interests are in the areas of simulation, applied statistics, and the applications of these techniques to emergency response. She is a member of INFORMS and IIE. Her e-mail address is $<$ ta27@buffalo.edu>. 
DAVID GOLDSMAN is a Professor in the School of Industrial and Systems Engineering at the Georgia Institute of Technology. He received his Ph.D. in Operations Research and Industrial Engineering from Cornell University. His research interests are in the areas of simulation, applied statistics, and applications of operations research in medical and humanitarian problems. He is a member of INFORMS and is the IIE Board Representative to the Winter Simulation Conference. His e-mail address is <sman@gatech. edu>.

JAMES R. WILSON is professor and head of the Edward P. Fitts Department of Industrial and Systems Engineering at North Carolina State University. He received his Ph.D. degree in industrial engineering from Purdue University. His current research interests are focused on the design and analysis of simulation experiments. He also has an active interest in applications of operations research techniques to all areas of industrial and systems engineering. He is currently the Editor-in-Chief of ACM Transactions on Modeling and Computer Simulation. He is a member of AAUW, ACM, and ASA, and he is a Fellow of IIE and INFORMS. His e-mail address is <jwilsonencsu.edu>, and his web page is <www. ise.ncsu. edu/jwilson>. 